\title{
RACIONALIDADE DAS DECISÕES ESTRATÉGICAS
}

\section{RATIONALITY IN THE STRATEGIC DECISIONS}

\section{LA RACIONALIDAD EN LAS DECISIONES ESTRATÉGICAS}

\section{José Emilio Navas López}

Doctor en Ciencias Económicas y Empresariales, Universidad Complutense de Madrid - España Licenciado en Ciencias Económicas y Empresariales, Universidad de Málaga

E-mail: jenavas@ccee.ucm.es (Espanha)

\section{Luis Ángel Guerras Martín}

Licenciado y Doctor en Ciencias Económicas y Empresariales por la Universidad Complutense de Madrid - España

E-mail: luisangel.guerras@ urjc.es (Espanha)

\section{Antonio Montero Navarro}

Profesor Titular de Escuela Universitaria de Organización de Empresas, Universidad Rey Juan Carlos. Facultad de Ciencias Jurídicas y Sociales - España

E-mail: antonio.montero@urjc.es (Espanha) 


\section{RACIONALIDADE DAS DECISÕES ESTRATÉGICAS}

\section{RESUMO}

O atual artigo tem o objetivo de analisar a conveniência ou não de que o processo de tomada de decisões seja mais ou menos racional. Em principio, este processo deveria responder a um esquema de uma rigorosa racionalidade de acordo com a lógica econômica. Porém, na realidade, este processo nem sempre ocorre assim, surgindo diversos fatores que contribuem a potencializar ou a reduzir essa racionalidade. Este trabalho propõe, em primeiro lugar, um modelo rigorosamente racional de decisão estratégica, a partir do qual se deduzem suas principais vantagens e pontos fortes, para a adoção das melhores decisões estratégicas. Posteriormente, se mencionam as críticas a esse modelo e se apresentam os fatores dos quais, na prática, depende a maior ou menor racionalidade do processo. A partir desta comparação, as conclusões defendem a utilização de um processo que se inicie de pressupostos racionais, embora sendo conscientes das diferentes influências internas e externas que afetarão o decorrer das decisões, reduzindo essa racionalidade.

Palavras-chave: Tomada de Decisão; Processo; Racionalidade; Modelo Racional.

\section{RATIONALITY IN THE STRATEGIC DECISIONS}

\section{ABSTRACT}

The goal of this paper is to analyze the relative convenience of using a rational process for making strategic decisions. According to traditional views, the strategic decision making process should be aligned with the so-called rational or synoptic process. Nevertheless, a look at the decisions taken by firms clarifies that this is far from the truth, -there are many aspects which could foster or refrain the degree of rationality. After explaining a wholly rational strategic process coupled with its potential strengths, this paper presents the principal criticisms to the model, in addition to the factors conditioning the level of rationality of the process in practice. As a consequence of this comparison, our conclusions defend the use of an intended rational process, bearing in mind the multiple internal and external influences that will affect the decision scheme, undermining the degree of rationality.

Keywords: Decision Making; Process; Rationality; Rational Model.

Revista Ibero-Americana de Estratégia - RIAE, São Paulo, v. 9, n. 1, p. 182-199, jan./abr. 2010. 
José Emilio Navas López, Luis Ángel Guerras Martín \& Antonio Montero Navarro

\section{LA RACIONALIDAD EN LAS DECISIONES ESTRATÉGICAS}

\section{RESUMEN}

El presente artículo tiene por objetivo analizar la conveniencia o no de que el proceso de toma de decisiones estratégicas sea más o menos racional. En principio, dicho proceso debería responder a un esquema de estricta racionalidad de acuerdo con la lógica económica. Sin embargo, en la realidad, este proceso no siempre ocurre así, apareciendo diversos factores que contribuyen a potenciar o a reducir dicha racionalidad. Este trabajo propone, en primer lugar, un modelo estrictamente racional de decisión estratégica, a partir del cual se infieren sus principales ventajas y puntos fuertes, para la adopción de las mejores decisiones estratégicas. Posteriormente, se plantean las críticas a dicho modelo y se exponen los factores de los que, en la práctica, depende la mayor o menor racionalidad del proceso. A partir de esta comparación, las conclusiones abogan por la utilización de un proceso que parta de supuestos racionales, aunque siendo conscientes de las diferentes influencias internas y externas que afectarán el curso de las decisiones, reduciendo dicha racionalidad.

Palabras-clave: Toma de Decisiones; Proceso; Racionalidad; Modelo Racional. 


\section{INTRODUCCIÓN: LA TOMA DE DECISIONES ESTRATÉGICAS}

Según los postulados de la economía neoclásica, las empresas toman sus decisiones de manera automática: ante la necesidad de introducir un cambio en la organización, reconocida por la empresa, ésta de inmediato pondrá en marcha una acción que le permita maximizar su objetivo, la obtención de beneficios o la creación de valor.

Un análisis detenido de este planteamiento nos conduce a diversas ideas, claramente alejadas de la realidad empresarial. Decidir consiste, desde este punto de vista, en un acto, esto es, algo instantáneo que no requiere la participación de ningún agente: ni directivos, ni órganos de asesoramiento, ni consultores están "invitados" a esta toma de decisiones. De hecho, estrictamente no podríamos hablar de decisiones, sino de actuaciones, dado que siempre se hace, de modo inmediato, lo mejor para la empresa. Los agentes económicos son, de esta forma, plenamente racionales, porque maximizan en todo caso sus objetivos, sean éstos los beneficios (empresas) o la utilidad (individuos).

Entre las diversas críticas a este planteamiento, hay que destacar la de Simon (1945), que abre una corriente de investigación en torno al proceso de toma de decisiones estratégicas que llega hasta el momento actual. Este autor planteó que, en un entorno real de decisión, no es posible disponer de toda la información ni conocer todas las alternativas, no es posible conocer a priori con certeza el resultado de una decisión antes de implantarla y no siempre se elige la alternativa que maximiza los resultados, sino que se opta por una alternativa que satisface suficientemente el logro de los objetivos propuestos.

Supongamos, por ejemplo, que una empresa dedicada a la hostelería quiere construir un nuevo complejo en una zona de alto interés turístico. En una primera selección, los responsables de la decisión consideran un número determinado de alternativas, ignorando en muchas ocasiones otras zonas con un potencial importante, que podrían haber conducido a un beneficio superior. Además, a la hora de valorar cada alternativa, aunque la experiencia permite establecer algunas expectativas de afluencia de turistas, existen factores que no pueden ser controlados a priori (un posible desastre natural, una evolución desfavorable del mercado de divisas, etc.) que podrían situar la rentabilidad real muy por debajo de la esperada. Finalmente, aunque se crea firmemente, apoyándose en valoraciones razonables, que una localización determinada generaría beneficios superiores a todas las demás alternativas, el poder de uno de los socios (o un grupo de socios) de la empresa, puede hacer que la localización elegida finalmente sea otra, a priori no tan atractiva.

Revista Ibero-Americana de Estratégia - RIAE, São Paulo, v. 9, n. 1, p. 182-199, jan./abr. 2010. 
José Emilio Navas López, Luis Ángel Guerras Martín \& Antonio Montero Navarro

Así, la visión de Simon (1945), lejos de defender la existencia de agentes suprahumanos que siempre saben lo que hay que hacer, plantea la necesidad de utilizar un proceso más o menos complejo que integra lo que podemos denominar "decisión”. En efecto, toda decisión empresarial parte del reconocimiento de la necesidad de decidir, lo cual ya es en sí mismo un problema. Posteriormente, es necesario buscar la información relativa a la decisión, analizarla, construir alternativas entre las que elegir y elegir entre estas alternativas (utilizando algún criterio). Finalmente, es preciso implantar la decisión tomada, con unas u otras consecuencias. Este proceso, con el fin de cuestionarlo, es detallado por Mintzberg, Raisinghani y Théorêt (1976) a través de un entramado de fases y rutinas. Así, no sólo importa qué decisión se toma, sino cómo se toma una decisión.

La utilización de esta visión teórica tiene también consecuencias lógicas. Podemos decir que este planteamiento es el que da verdadero origen a la dirección de empresas, por cuanto tiene que haber agentes que conduzcan todo este proceso, tomen parte en sus diferentes fases y, sobre todo, seleccionen la alternativa a implantar. En este sentido, una decisión no será más o menos racional por su resultado (mayores o menores beneficios), sino en función de cómo se ha tomado, esto es, de la utilización de un proceso más o menos estructurado para llegar a ella.

Partiendo de la descripción de una visión tradicional de estricta racionalidad de los procesos de toma de decisiones estratégicas, este trabajo recoge las principales reflexiones y críticas sobre la utilidad de dicho modelo en un contexto económico como el actual, cuestionada por modelos que definen el nivel de racionalidad de los procesos de toma de decisiones estratégicas, en función de diversas variables referentes al entorno, la decisión o la propia empresa.

Con el fin de realizar esta reflexión, se han estudiado algunos de los artículos de referencia en el análisis del proceso de toma de decisiones estratégicas, que permiten una cierta revisión de la literatura sobre el tema. Así, en el apartado siguiente se estudia con profundidad el modelo racional de decisión estratégica tanto para describirlo como para destacar sus ventajas y las críticas que ha recibido. El tercer apartado se centra en determinar los factores que influyen en que las decisiones adoptadas en la empresa sean más o menos racionales. El artículo termina con las principales ideas desarrolladas a modo de reflexión y conclusión.

Revista Ibero-Americana de Estratégia - RIAE, São Paulo, v. 9, n. 1, p. 182-199, jan./abr. 2010. 


\section{EL MODELO RACIONAL DE DECISIÓN ESTRATÉGICA}

La dirección actual de la empresa se enfrenta a un entorno cada vez más cambiante, complejo y hostil, lo que hace necesario abordar el tipo de respuesta que la empresa debe dar para sobrevivir o para aumentar su eficacia y eficiencia en estas circunstancias. La solución a esta necesidad es lo que se pretende a través de la elección de la llamada estrategia empresarial que, a su vez, surge de un complejo conjunto de actividades que denominaremos el proceso de dirección estratégica.

La dirección estratégica no es algo distinto de la dirección de empresas, si bien podemos precisar que es aquella parte de la dirección empresarial que tiene como objetivo primordial formular una estrategia y ponerla en práctica. La complejidad del problema que se plantea con la adopción de una decisión estratégica y su puesta en marcha, tiene la suficiente magnitud como para demandar la utilización de una metodología apropiada. Existen diversas aportaciones a este respecto, cuya finalidad es poner de manifiesto las distintas actividades a realizar a la hora de definir e implantar, posteriormente, una solución estratégica. En casi todas ellas se hace referencia, de acuerdo con lo expuesto anteriormente, a la idea de la dirección estratégica como un proceso, una secuencia de fases o actividades que se realizan en el tiempo, de acuerdo con un orden determinado. Una visión de síntesis de dichas aportaciones aparece recogida en la figura 1.

Podemos considerar que este proceso global de la dirección estratégica de la empresa está integrado por tres grandes partes: análisis estratégico, formulación de estrategias e implantación de estrategias. Si bien existe un orden lógico en el desarrollo temporal de las partes básicas, el proceso se concibe de forma más realista a partir de la interacción constante de los distintos elementos incluidos en cada una de las partes o bloques, por lo que se hace necesaria una realimentación de información constante a lo largo del proceso (representada en la figura 1 por las líneas de puntos).

Podemos entender por "análisis estratégico" de una empresa, el proceso mediante el cual es posible determinar el conjunto de amenazas y oportunidades que el entorno presenta a la organización, así como el conjunto de fortalezas y debilidades de la misma, de forma que permita a la dirección un diagnóstico y evaluación de la situación y la correspondiente formulación de una estrategia, una vez definidos la misión, la visión y los objetivos estratégicos de la empresa.

Con esta finalidad, el análisis estratégico se compondrá esencialmente del estudio de tres elementos que conjuntamente determinan el espacio estratégico en el que se va a desenvolver la empresa y que serán los ejes sobre los que se construirá su estrategia: la definición de los fines y misiones generales de la empresa, el análisis externo y el análisis interno.

Revista Ibero-Americana de Estratégia - RIAE, São Paulo, v. 9, n. 1, p. 182-199, jan./abr. 2010. 
José Emilio Navas López, Luis Ángel Guerras Martín \& Antonio Montero Navarro

Figura 1 - El proceso de dirección estratégica.

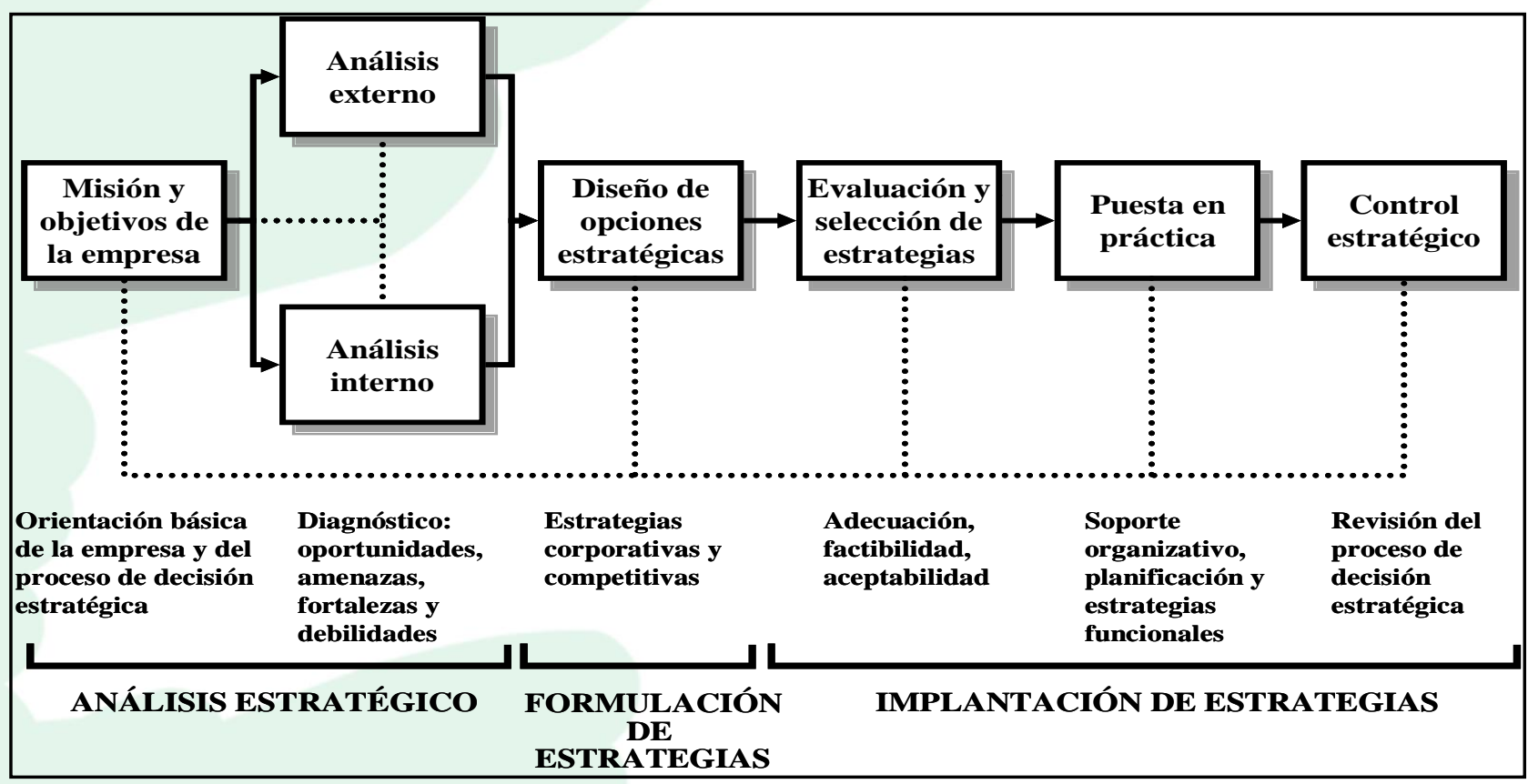

Fuente: Guerras y Navas (2007).

El segundo bloque de la dirección estratégica corresponde a lo que se denomina la "formulación de estrategias". A partir del análisis estratégico, y según la metodología propuesta, la empresa trataría de formular o diseñar - tanto al nivel de negocio como corporativo y funcional las posibles alternativas que se tienen para conseguir la misión y los objetivos que se han elegido a partir del contexto definido en los análisis externo e interno.

Una vez que han sido diseñadas diversas alternativas, es preciso evaluarlas, mediante la utilización de diferentes criterios, de modo que pueda ser elegida una de ellas para su implantación posterior. Los criterios que se utilicen para la evaluación de estrategias deben ser capaces de identificar la que, al menos a priori, parece la mejor estrategia o alternativa posible.

La última parte del proceso supone poner en marcha la estrategia elegida y recibe el nombre de "implantación estratégica". Para ello, es útil la elaboración de un plan estratégico que recoja los objetivos y las decisiones que se han adoptado en etapas anteriores, así como la asignación de medios y el camino a seguir para que la estrategia tenga éxito. Pero no todo depende de lo bien perfilado que esté el camino sino, entre otros factores, de la capacidad del equipo directivo para liderar y estimular la actividad de los recursos humanos de modo que los objetivos sean conseguidos efectivamente, así como de la estructura organizativa y la cultura empresarial que sirvan de soporte a la implantación.

Revista Ibero-Americana de Estratégia - RIAE, São Paulo, v. 9, n. 1, p. 182-199, jan./abr. 2010. 
Este proceso es llevado a cabo, al menos sobre el papel, por las empresas y organizaciones que realizan un esfuerzo explícito de planificación estratégica. Así, cuando una organización decide elaborar su Plan Estratégico, tras una primera reflexión sobre sus objetivos e identidad, se procede a una búsqueda de información sobre el entorno en el que actúa, así como sobre la propia realidad de la organización: los recursos de los que dispone, su cultura, sus relaciones con otros agentes, etc. Posteriormente, con la información de que se dispone, los responsables de la elaboración del Plan Estratégico trabajan en el establecimiento de una serie de ámbitos genéricos y líneas específicas de actuación que van a configurar la estrategia de la organización en los siguientes años. Finalmente, cada actuación es encuadrada en un horizonte temporal, habitualmente vinculado a una programación tanto temporal como de los medios necesarios para poner en marcha el plan establecido.

\subsection{VENTAJAS DEL MODELO RACIONAL}

El modelo representado en la figura 1 responde a un proceso "racional" o "sinóptico" y “deliberado" o "intencional” de tomar decisiones estratégicas. En un proceso de este tipo se asume que se realiza una búsqueda extensiva de informaciones, se diseña en profundidad un número razonable de alternativas y se evalúan dichas alternativas con criterios objetivos. Este modelo ha sido defendido en ocasiones como un "proceso ideal", beneficioso para la empresa.

Asimismo, es el enfoque seguido por la mayor parte de los investigadores teóricos, debido a su alta capacidad explicativa y al hecho de que, en la mayor parte de los casos, supone de algún modo una base para explicar cualquier otro tipo de proceso. Las principales ventajas que presenta esta aproximación son (Wheelen \& Hunger, 2002; David, 2003; Thompson \& Strickland, 2004):

- Facilita un análisis más sistemático, lógico y racional de la decisión que se deba adoptar, obligando a los directivos a buscar, elaborar y analizar información para mejorar la calidad de las decisiones estratégicas. Ello permite, entre otras cosas, identificar las cuestiones importantes para el futuro de la empresa, ser más consciente de las amenazas y oportunidades del entorno y de las fortalezas y debilidades internas, pudiendo de esta manera centrar la atención de la organización en ellas.

Revista Ibero-Americana de Estratégia - RIAE, São Paulo, v. 9, n. 1, p. 182-199, jan./abr. 2010. 
José Emilio Navas López, Luis Ángel Guerras Martín \& Antonio Montero Navarro

- Permite a la empresa ser más proactiva que reactiva al definir su propio futuro. Es decir, la empresa puede tratar de influir en el entorno y en su evolución y no simplemente esperar los cambios del entorno para reaccionar antes de que sea demasiado tarde.

- Facilita la comprensión a todos los miembros de la organización de lo que la empresa quiere hacer y, como consecuencia de ello, aumenta el compromiso y contribuye a mejorar la implantación. Una clara definición de la misión y los objetivos puede ser útil en este aspecto.

- Ayuda a evaluar las decisiones menos estratégicas que toman los directivos, al facilitar su comparación con la estrategia elegida y la orientación general de la empresa. De esta forma, se facilita una coherencia y coordinación entre las múltiples decisiones que los directivos deben tomar constantemente.

- Facilita la evaluación de la marcha de la estrategia y, por tanto, la identificación de problemas en el desarrollo de la estrategia o de potenciales fuentes de problemas en el futuro.

- Permite la participación de más personas en el proceso de dirección estratégica, al establecer un marco claro de referencia para el análisis y las decisiones. Esto posibilita la incorporación de directivos de nivel medio o inferior al proceso que puedan mejorar la calidad de las decisiones.

Como consecuencia de las anteriores ventajas, cabría esperar que las empresas que sigan un proceso racional obtengan mejores resultados, por lo que, en principio, todas tenderían a emplearlo. Sin embargo, la realidad muestra que las empresas utilizan en ocasiones diferentes esquemas de decisión, ya que el seguimiento del proceso racional no garantiza el éxito de la estrategia. De hecho, como han llegado a demostrar algunos estudios, la utilización de un proceso plenamente racional puede llegar a ser contraproducente, empeorando los resultados de la empresa (Fredrickson \& Mitchell, 1984).

Revista Ibero-Americana de Estratégia - RIAE, São Paulo, v. 9, n. 1, p. 182-199, jan./abr. 2010. 
Así, del mismo modo que el estudio del proceso de toma de decisiones partió de una crítica "realista" al modelo neoclásico, pronto comenzaron a surgir visiones que cuestionaban el grado de realismo de los procesos "racionales", proponiendo en algunos casos modelos alternativos.

\subsection{CRÍTICAS AL MODELO RACIONAL}

Se podría afirmar que la mayor parte de las críticas al proceso racional o sinóptico se basan en su excesiva "asepsia". En efecto, su planteamiento inicial, aunque más inclusivo que el modelo neoclásico, olvida que las actividades de una empresa se desarrollan en un contexto interno (la propia empresa) y externo (su entorno), caracterizado por su complejidad, incertidumbre y conflicto.

Así, en la realidad empresarial, en muchas ocasiones, los objetivos no están claros y cambian a lo largo del tiempo; las personas a menudo buscan la información y las alternativas de forma desordenada y oportunista; el análisis de alternativas puede estar limitado; y las decisiones a menudo reflejan más la utilización de procedimientos operativos estandarizados que un análisis sistemático y racional (Eisenhardt \& Zbaracki, 1992). Este hecho se traduce en la utilización de formas y procesos de decisión estratégica que podemos considerar alejados de una estricta racionalidad.

Algunos ejemplos de prácticas que divergen de la utilización de procesos sinópticos o racionales son la evaluación de alternativas de forma paralela o incluso anterior a su completa formulación. Otros son la búsqueda de información y hasta la evaluación del impacto económico de una alternativa estratégica que ya ha sido elegida para su implantación; la contratación del análisis estratégico a consultores externos para que confirmen la información ya conocida y valorada, buscando objetivar la decisión, o la "vuelta atrás" en procesos que han alcanzado una situación política excesivamente conflictiva y difícil de resolver sin un replanteamiento del problema.

El propio Simon (1945) abrió el camino a la crítica al proceso racional, poniendo de manifiesto cómo, dada la racionalidad limitada del decisor, el proceso de decisión estratégica no es lineal sino que, en la práctica, se producen ciclos de realimentación en los cuales los objetivos son reajustados conforme el proceso avanza. La cantidad de ciclos introducidos y la forma final del proceso dependerán de la mayor o menor complejidad de la decisión estratégica a adoptar y del mayor o menor conflicto de objetivos existente en la organización (Eisenhardt \& Zbaracki, 1992).

A partir de esta primera visión crítica, se han planteado con mayor o menor éxito diferentes visiones del proceso de toma de decisiones estratégicas cuestionando, por diferentes motivos, el realismo y utilidad del modelo racional o sinóptico.

Revista Ibero-Americana de Estratégia - RIAE, São Paulo, v. 9, n. 1, p. 182-199, jan./abr. 2010. 
José Emilio Navas López, Luis Ángel Guerras Martín \& Antonio Montero Navarro

- El incrementalismo lógico (Quinn, 1980) trata de resaltar la importancia del aprendizaje en el proceso de decisión estratégica. Este autor concibe una decisión estratégica como un proceso fragmentado que se da dentro de las distintas unidades organizativas a medida que se adaptan de forma reactiva a un entorno cambiante. Se inicia así un proceso de implantación parcial de la estrategia, mediante la "prueba y el error" a partir del cual la empresa pueda ir mejorando su conocimiento sobre los resultados de la aplicación. Esto permite a la empresa aprender de la experiencia y mejorar su conocimiento sobre la adecuación de la estrategia y, en último término, aumentar su capacidad de decisión. Sin embargo, también puede conducir a la ineficiencia de modo que distintas unidades de la organización empujen hacia objetivos y direcciones diferentes.

De este modo, no es infrecuente en las empresas la utilización de "programas piloto", que permitan valorar en un entorno controlado (una planta, una región, un área), como si se tratase de un laboratorio, la aplicación de políticas y/o estrategias que, tras un proceso de refinamiento, pueden pasar a ser utilizadas por el conjunto de la compañía.

- Visiones políticas del proceso (Cyert \& March, 1965). Haciendo especial hincapié en el contexto interno de las decisiones, diversos autores sostienen desde la Teoría de la Organización, que las organizaciones están formadas por personas y grupos (stakeholders) que tienen diferentes objetivos y preferencias que son, en buena medida, incompatibles entre sí, por lo que entran en conflicto. Si esto es así, el proceso de decisión estratégica no es algo aséptico y objetivo por definición, sino que tiene un carácter esencialmente político en el que cada grupo trata de conseguir sus objetivos, para lo cual se implica en actividades y tácticas políticas tales como la formación de coaliciones, la cooptación, el uso estratégico de la información, el recurso a consultores externos, etc. En general, el resultado del proceso de decisión responderá a los objetivos y deseos de los grupos con mayor poder dentro de la organización (Eisenhardt \& Zbaracki, 1992). Especialmente importante es el papel de los directivos que pueden influir de manera notable en el desarrollo del proceso (Guerras, 2004).

- El enfoque de garbage can o del cubo de la basura (Cohen, March \& Olsen, 1972). Estos autores han planteado cómo en ocasiones las organizaciones se asemejan más a 
anarquías organizativas, en cuyo seno convive un conjunto de agentes a los que, en un momento dado, se presentan una serie de decisiones que deben afrontar influidos por muchos factores. En este contexto, el azar aparece como una fuerza fundamental: en ocasiones la práctica muestra cómo un acontecimiento casual (una persona que llegue tarde a una reunión por un pequeño accidente, por ejemplo) puede hacer que se tome una decisión diferente a la que se hubiera adoptado en otras condiciones.

Por tanto, la realidad de un proceso de decisión estratégica se entiende mejor a partir de la complementariedad entre los aspectos racionales y las aportaciones de los distintos enfoques que sugieren aspectos menos racionales como los que se derivan de las razones analizadas. La figura 2 recoge los distintos enfoques del proceso de decisión estratégica.

\section{FACTORES QUE AFECTAN A LA RACIONALIDAD DEL PROCESO}

Además, hay que tener en cuenta que los procesos de decisión estratégica no se producen de manera uniforme en cada situación dentro de la organización ni, por supuesto, entre organizaciones diferentes. Así, las características específicas que adopte finalmente el modelo dependerán, en cada organización, de diversos tipos de factores, entre los que cabe destacar (Rajagopalan, Rasheed \& Datta 1993):

- El contexto interno de una organización, en el que tiene un peso especial el equipo ejecutivo (su composición, antigüedad, permanencia e incluso perfil psicológico) (Dahli, Weigart \& Hinds, 2005; Miller, Burke \& Glick, 1998), aunque también son relevantes los directivos de nivel medio, el Consejo de Administración progresivamente más involucrado en la estrategia de la empresa, como indica Rindova (1999) - o la estructura organizativa de la empresa.

- La naturaleza de la propia decisión estratégica a adoptar, tanto en lo que se refiere a sus características genéricas como a su contenido. Dos de los factores más influyentes en este ámbito son el origen de la decisión (una amenaza o una oportunidad) y el previsible impacto estratégico de dicha decisión (Schilit \& Payne, 1987).

Revista Ibero-Americana de Estratégia - RIAE, São Paulo, v. 9, n. 1, p. 182-199, jan./abr. 2010. 
José Emilio Navas López, Luis Ángel Guerras Martín \& Antonio Montero Navarro

Figura 2 - Diferentes enfoques del proceso de decisión estratégica.

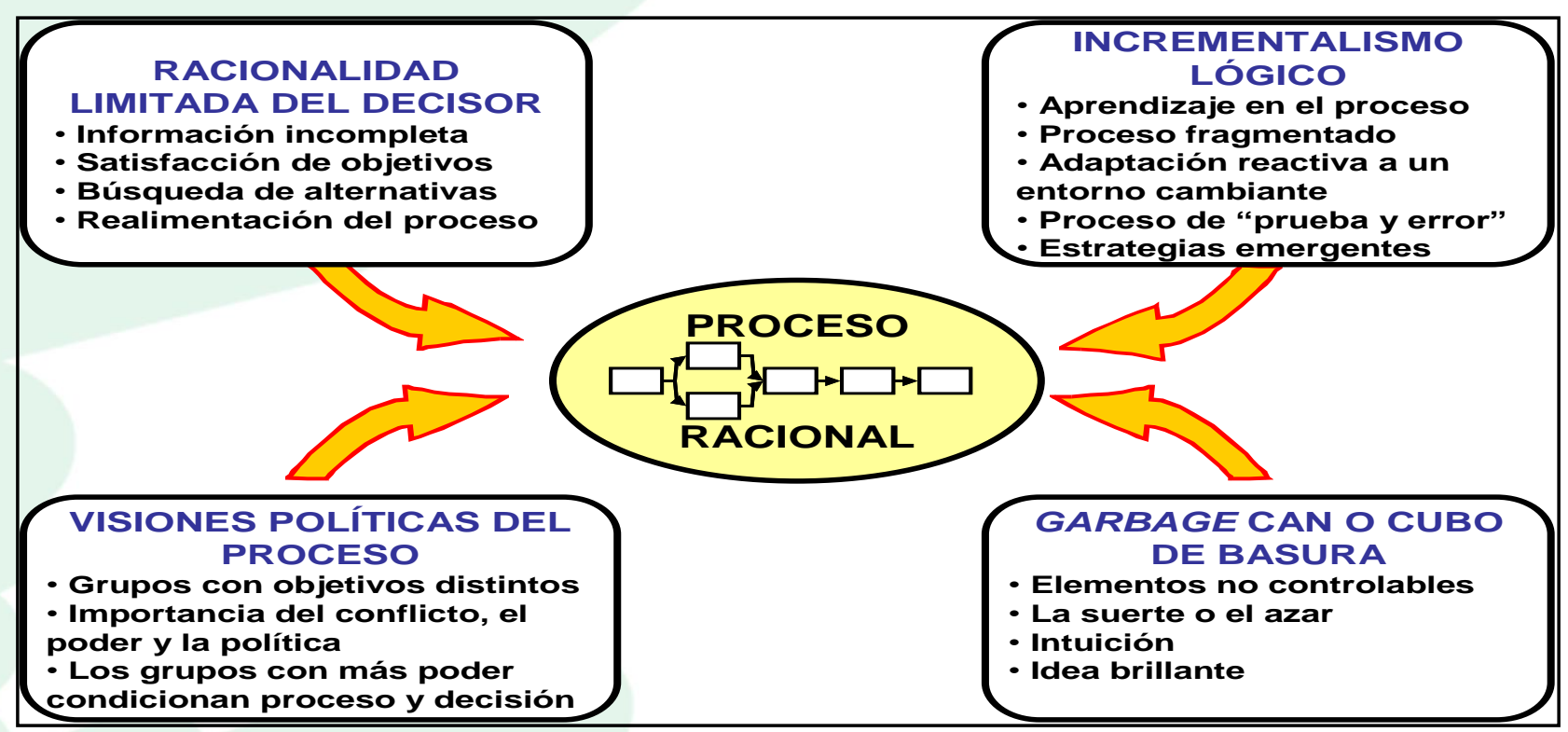

Fuente: Adaptada de Guerras y Navas (2007).

- El entorno de la empresa, caracterizado por una mayor o menor presión competitiva, la influencia en la decisión de agentes externos o un grado determinado de hostilidad. Todos ellos condicionan la forma de tomar las decisiones (Yasai Ardekani \& Haug, 1997; Kim, Payne \& Tan, 2006).

En general, podemos afirmar que los procesos de decisión estratégica nunca son totalmente racionales ni totalmente políticos, siendo una cuestión de grado el que se sitúen más o menos cerca de cada uno de los extremos. Algunos de los factores que favorecen o perjudican el carácter racional del proceso de decisión estratégica (Figura 3) son los siguientes (Dean \& Sharfman, 1993):

- Amenaza competitiva: cuando la competencia es más intensa y el entorno más hostil, las decisiones adoptadas pueden tener consecuencias graves para la organización, por lo que el nivel de racionalidad del proceso tiende a aumentar.

- Importancia de la decisión: cuando la decisión estratégica que debe ser adoptada sea más importante, comprometa en mayor medida el futuro de la empresa y las consecuencias de un acierto o error sean más decisivas, la dirección tenderá a analizar con mayor profundidad las decisiones en este contexto.

Revista Ibero-Americana de Estratégia - RIAE, São Paulo, v. 9, n. 1, p. 182-199, jan./abr. 2010. 
- Control externo: si existen mecanismos de control externo de las decisiones adoptadas, los directivos harán un mayor esfuerzo por mejorar la calidad de la decisión, por lo que utilizarán más información y técnicas de análisis. Los accionistas, el mercado de capitales o de empresas pueden ser mecanismos externos que influyan en la dirección descrita.

- Conflicto de objetivos: la existencia de diversidad o conflicto de objetivos entre los grupos participantes en la organización o entre el propio equipo de alta dirección hará que el proceso sea menos racional y pueda darse un mayor contexto político.

- Incertidumbre: cuanto mayor sea la incertidumbre que rodea el proceso de elección estratégica menor será el grado de racionalidad del mismo. Ello se debe a que la posibilidad de aplicar modelos de análisis y evaluación racionales es menor, por lo que tiende a confiarse más en la intuición, el juicio o la tradición.

- Tamaño de la organización: el mayor tamaño de una empresa hace que las decisiones estratégicas que deban ser adoptadas sean más complejas por lo que aumenta la dificultad para aplicar métodos racionales de decisión.

Sin embargo, ni en este ni en otros trabajos existe evidencia empírica definitiva para demostrar la influencia de los distintos factores en la forma descrita. Además, el estudio de la relación entre la racionalidad de los procesos de toma de decisiones estratégicas y la rentabilidad empresarial arroja resultados contradictorios: en ocasiones los procesos más racionales obtienen mejores resultados, pero no siempre. 
José Emilio Navas López, Luis Ángel Guerras Martín \& Antonio Montero Navarro

Figura 3 - Determinantes de la racionalidad de los procesos de decisión estratégica.

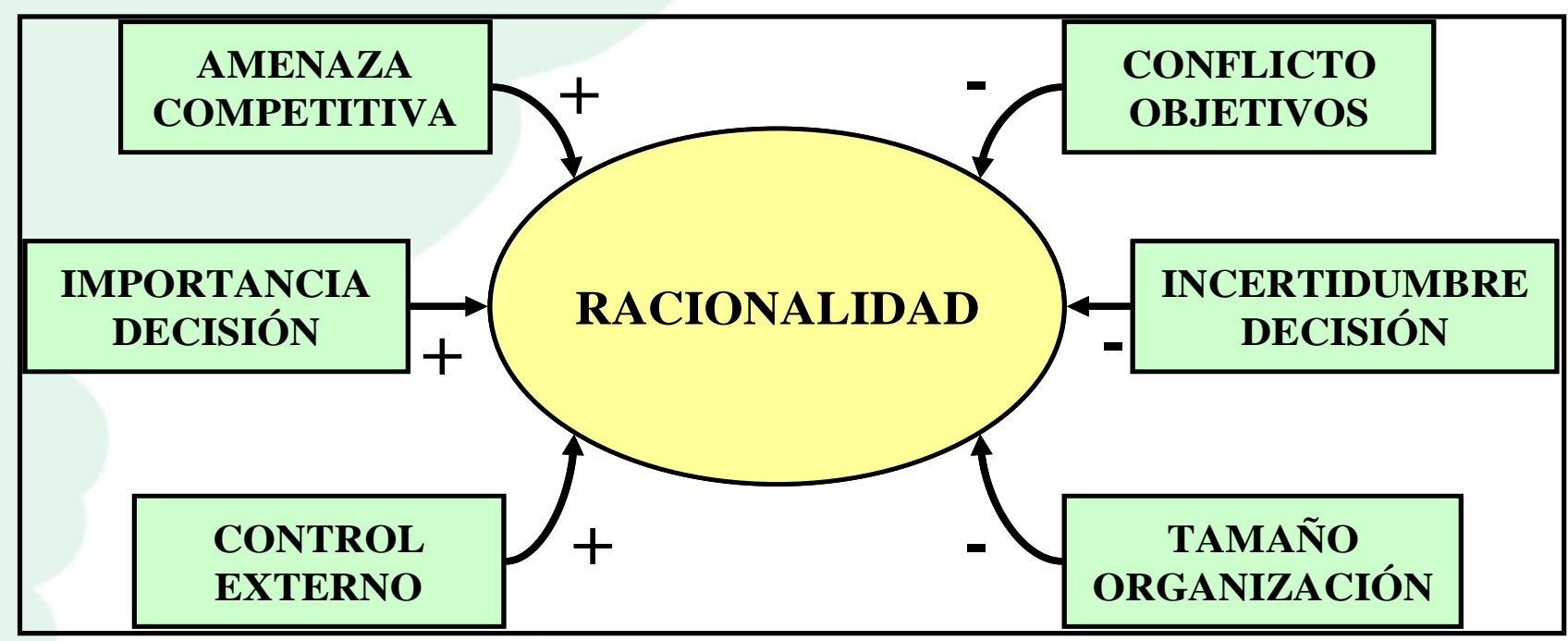

Fuente: Guerras y Navas (2007).

Finalmente, es preciso indicar que la racionalidad no es el único elemento a valorar en un proceso de toma de decisiones. Algunas características como la rapidez de la decisión, el nivel y tipo de conflicto generado en el proceso o la percepción de justicia procesal pueden guardar también relación con los resultados de la empresa. Estos factores pueden tener, además, ciertos efectos de compensación (trade off) con la racionalidad del proceso: en la práctica, el diseño de un proceso que permita tomar decisiones altamente racionales, rápidas, con un nivel adecuado de conflicto cognitivo y no afectivo (Amason, 1996) y que sean percibidas como justas por los participantes sería la verdadera piedra filosofal de la dirección estratégica.

\section{CONCLUSIONES}

Los modelos de dirección estratégica han respondido tradicionalmente a un esquema de decisiones racionales en el que se busca permanentemente la mejor decisión posible dentro de una lógica económica. Es decir, las empresas buscan optimizar los resultados a partir de una situación o contexto determinado. Sin embargo, en la realidad, diversos factores dificultan este proceso de decisión racional, introduciendo nuevos elementos que modifican o matizan la visión más racionalista.

Entre estos factores aparece la incertidumbre, la complejidad y el conflicto que rodean a las decisiones estratégicas y que condicionan la forma de tomarlas. Como consecuencia de ello, se han

Revista Ibero-Americana de Estratégia - RIAE, São Paulo, v. 9, n. 1, p. 182-199, jan./abr. 2010. 
planteado enfoques alternativos para describir el proceso de toma de decisiones estratégicas tales como la racionalidad limitada del decisor, el incrementalismo lógico, la visión política o el enfoque del cubo de basura. Todos ellos aportan visiones alternativas al proceso lógico y enriquecen el conocimiento que tenemos del proceso de decisiones estratégicas.

En la práctica, y como conclusión, podemos señalar que ambos aspectos - el racional y el menos racional - son como dos caras de una misma moneda, dos partes complementarias de una misma realidad. Un proceso de decisión estratégica que no atendiera suficientemente a un enfoque racional podría incurrir fácilmente en una especie de suicidio de la empresa, al no tener en cuenta el análisis de las mejores opciones desde el punto de vista económico. Sin embargo, en muchas situaciones sería ingenuo pensar que aspectos relacionados con el poder o con las influencias o incluso el azar, no tienen que ver con algunos de los éxitos o fracasos estratégicos de la historia empresarial.

De esta forma, la cuestión no es tanto si el proceso debe ser racional o no, sino cómo entender los factores racionales y los menos racionales y tratar de dirigir la empresa equilibrando la influencia de ambos tipos de factores en cada momento. La importancia que dicha influencia tenga va a depender, además, de algunas variables clave tales como la amenaza competitiva, la importancia de la decisión adoptada o la incertidumbre de la decisión, entre otros.

\section{REFERENCIA}

Amason, A. (1996). Distinguishing the effects of functional and dysfunctional conflict on strategic decision making: Resolving a paradox for top management teams. Academy of Management Journal, 39 (1), 123-148. http://dx.doi.org/10.2307/256633

Cohen, M. D., March, J. G., Olsen, J. P. (1972). A garbage can model of organizational choice, Administrative Science Quarterly, 17 (1), 1-25.

http://dx.doi.org/10.2307/2392088

Cyert, R., March, J. (1965). Teoría de las decisiones económicas en la empresa. Englewood Cliffs: Prentice Hall.

Dahlin, K. B., Weingart, L. R., Hinds, P. J. (2005). Team diversity and information use. Academy of Management Journal, 48 (6), 1107-1123. http://dx.doi.org/10.5465/AMJ.2005.19573112

Revista Ibero-Americana de Estratégia - RIAE, São Paulo, v. 9, n. 1, p. 182-199, jan./abr. 2010. 
José Emilio Navas López, Luis Ángel Guerras Martín \& Antonio Montero Navarro

David, F. R. (2003). Conceptos de administración estratégica (9a ed.). México: Pearson.

Dean, J. W., Sharfman, M. P. (1993). Procedural rationality in the strategic decision-making process. Journal of Management Studies, 30 (4), 587-610.

http://dx.doi.org/10.1111/j.1467-6486.1993.tb00317.x

Eisenhardt, K. M., Zbaracki, M. J. (1992). Strategic decision making. Strategic Management Journal, 13 (número especial invierno), 17-37.

Fredrickson, J., Mitchell, T. (1984). Strategic decision processes: Comprehensiveness and performance in an industry with an unstable environment. Academy of Management Journal, 27 (2), 399-423.

http://dx.doi.org/10.2307/255932

Guerras Martín, L. A. (2004). Problemas organizativos en el proceso de la dirección estratégica. Universia Business Review, 1, 116-125.

Guerras Martín, L. A., Navas López, J. E. (2007). La dirección estratégica de la empresa. Teoría y aplicaciones (4a ed.). Madrid: Thomson-Civitas.

Kim, K. H., Payne, G. T., Tan, J. A. (2006). An examination of cognition and affect in strategic decision making. International Journal of Organizational Analysis, 14 (4), 277-295.

http://dx.doi.org/10.1108/19348830610849709

Miller, C., Burke, L., Glick, W. (1998). Cognitive diversity among upper echelon executives: Implications for strategic decision processes. Strategic Management Journal, 19 (1), 39-58. http://dx.doi.org/10.1002/(SICI)1097-0266(199801)19:1<39::AID-SMJ932>3.0.CO;2-A

Mintzberg, H., Raisinghani, D., Théorêt, A. (1976). The structure of unstructured decision processes. Administrative Science Quarterly, 21 (2), 246-275.

$\underline{\text { http://dx.doi.org/10.2307/2392045 }}$

Quinn, J. (1980). Strategies for change: Logical incrementalism. Homewood, Illinois: Irwin.

Rajagopalan, N., Rasheed, A., Datta, D. (1993). Strategic decision processes: Critical review and future directions. Journal of Management, 19 (2), 349-384.

http://dx.doi.org/10.1177/014920639301900207

http://dx.doi.org/10.1016/0149-2063(93)90057-T

Rindova, V. (1999). What corporate boards have to do with strategy: A cognitive perspective. Journal of Management Studies, 36 (7), 953-975.

http://dx.doi.org/10.1111/1467-6486.00165

Revista Ibero-Americana de Estratégia - RIAE, São Paulo, v. 9, n. 1, p. 182-199, jan./abr. 2010. 
Schilit, W., Payne, F. (1987). An examination of the underlying dynamics of strategic decisions subject to upward influence activity. Journal of Management Studies, 24 (2), 161-181. http://dx.doi.org/10.1111/j.1467-6486.1987.tb00942.x

Simon, H. A. (1945). Administrative behavior, Nueva York, MacMillan [editada en castellano en 1978 con el título "El Comportamiento Administrativo", Aguilar, Madrid].

Thompson, A. A., Strickland, A. J. (2004). Administración estratégica: Textos y casos (13a ed.). México: McGraw-Hill Interamericana.

Wheelen, T. L., Hunger, J. D. (2002). Concepts in strategic management and business policy (8a ed.). Upper Saddle River: Prentice-Hall.

Yasai Ardekani, M., Haug, R. (1997). Contextual determinants of strategic planning processes. Journal of Management Studies, 34 (5), 729-767. http://dx.doi.org/10.1111/1467-6486.00070

Recebido: 08/01/2010

Aprovado: 15/03/2010

Revista Ibero-Americana de Estratégia - RIAE, São Paulo, v. 9, n. 1, p. 182-199, jan./abr. 2010. 